\title{
La representación del fenómeno migratorio en la prensa escrita de Tenerife
}

Elaborado por Daniel Buraschi e Inmaculada Fumero de LeÓN

Los medios de comunicación, y su importancia en la definición del discurso que cala en la sociedad, son el hilo conductor de este texto que analiza, cuantitativa y cualitativamente, el tipo de tratamiento y el encuadre que se elabora para las noticias de las llegadas de personas a Tenerife. 


\section{Introducción: medios de comunicación e inmigración}

Del conocimiento y la comprensión de la representación del fenómeno migratorio en los medios de comunicación, podemos deducir elementos clave en relación a las opiniones y actitudes de la ciudadanía respecto a este fenómeno.

Los medios de comunicación tienen la capacidad de enfocar el interés del público en cuestiones determinadas, al tiempo que de modo especular, excluir determinados eventos. La agenda de los medios, entendida como el conjunto de temas que se consideran de interés, influye a su vez en la agenda política y en la social. Esto es: por un lado guía el foco de atención del público, resaltando algunos temas y excluyendo otros; por el otro, fija el grado de relevancia de un determinado tema.

Las migraciones irregulares por vía marítima, por ejemplo, a pesar de no ser la principal vía de entrada de la inmigración irregular, representan uno de los temas más recurrentes en los medios cuando se habla de los fenómenos migratorios (Igartua, Muñiz y Cheng, 2005; Van Dijk, 2016). Así, se sobredimensiona en la opinión pública la intensidad, el volumen y el impacto de las migraciones marítimas.

Es decir, debemos tener en cuenta no sólo lo que se dice, sino también lo que no se dice. Los medios de comunicación pueden contribuir a invisibilizar el importante papel que desempeñan las personas migrantes en nuestra sociedad.

Además de definir los temas relevantes, los medios de comunicación ofrecen siempre, explícita o implícitamente, un determinado encuadre de la noticia; esto es, que la noticia vehicula determinados valores, creencias, y expectativas, que influyen en los destinatarios. Encuadrar una noticia significa enfocar un tema de una forma determinada; fijar una agenda de atributos; seleccionar las expresiones clave que definen el discurso; dotar de énfasis a algunas de estas expresiones; y definir una "idea organizadora central" para construir la historia informativa.

En este contexto, los investigadores definen como framing la capacidad de los medios de comunicación para provocar determinadas conclusiones en la audiencia, según la forma en que presentan la información. Los mismos hechos noticiosos pueden presentarse de forma diferente $y$, a veces contradictoria. Podemos destacar lo positivo o lo negativo, evidenciar el papel activo de las personas migrantes o representarlos a través de esterotipos y como un grupo homogéneo y pasivo; definiéndolos desde la alteridad.

Los estudios llevados a cabo en Europa, destacan que la representación mediática de la inmigración es predominantemente negativa. Los temas que se destacan son los problemas de legalidad de los inmigrantes, las diferencias culturales (el fundamentalismo religioso) y los problemas de relación interétnica (Van Dijk, 2016). También los estudios llevados a cabo en España destacan que el encuadre dominante de la inmigración es negativo (Igartua, Muñiz y Cheng, 2005). Además, existe una escasa variedad temática y una falta de contextualización en este tipo de noticias, en especial en la televisión (Igartua y Muñiz, 2004).

En un análisis de los encuadres noticiosos sobre inmigración llevado a cabo por Igartua, Muñiz y Cheng (2005), se identificaron los siguientes encuadres dominantes: "entrada irregular de inmigrantes en pateras", "actuación sobre menores inmigrantes", "los inmigrantes viven en condiciones de miseria, sufren desamparo y necesitan ayuda" o "los inmigrantes como actores conflictivos que protagonizan incidentes, motines, ataques y fugas". 
Más recientemente Fajardo Fernández y Soriano Miras (2016: 142), a partir del análisis de las noticias publicadas en seis periodicos nacionales y tres regionales, destacan que "los migrantes son retratados en la mayoría de los casos como agentes pasivos, como víctimas de las mafias o como objeto de atención asistencial por parte de diferentes entidades. En algunas noticias aparecen como actores que hacen uso de la violencia para alcanzar su propósito, mientras que son pocos los casos en los que aparecen como sujeto activo no asociado a conflictos. En suma, el relato dominante 'desciudadaniza' a estas personas en los distintos discursos analizados".

Sin embargo, si bien existe una tendencia general hacia los encuadres temáticos centrados en la inmigración irregular y a la vinculación entre inmigración y problemas sociales, hay que tener en cuenta que la investigación sobre este tema ha destacado que existen diferencias notables según la orientación ideológica del periódico, el colectivo de inmigrantes del cual se habla, y el contexto migratorio y político.

El tipo de encuadre dominante no depende solo de la orientación ideológica de periódicos, existiendo marcadas diferencias entre periódicos de derechas y de izquierdas, sino también entre periódicos nacionales y locales. Por ejemplo, en un estudio de Fernández y Corral (2016), han comprobado que en los diarios regionales se hacía hincapié en aspectos relacionados con lo laboral, cultural y convivencial, mientras que en los periódicos nacionales publicados en España se hacía una mayor referencia a los conflictos generados.

En Canarias, existen pocos análisis sistemáticos del tratamiento informativo de la inmigración. Los estudios empíricos se centran, sobre todo, en los años de la "crisis de los cayucos" (2006-2008). Rodríguez Borges (2010) ha llevado a cabo un amplio estudio de las noticias publicadas a lo largo de 2006 en cinco periódicos canarios (El Día, El Diario de Avisos, La Opinión, La Provincia y Canarias 7). El análisis del contenido temático de los artículos seleccionados, evidencia que la gestión de los flujos migratorios irregulares (llegadas, retenciones, traslados y repatriaciones) y las polémicas entre partidos políticos e instituciones, son el aspecto dominante en el 79,2\% de las noticias sobre inmigración.

Estos subtemas principales se corresponden con las fuentes informativas más utilizadas (instituciones oficiales y partidos políticos), confirmando que la naturaleza de las fuentes, predetermina qué facetas de la realidad inmigratoria aparecerán reflejadas en las informaciones. Además, se hace referencia a la presencia, en algunos periódicos, de una línea editorial que hacía explícitamente hincapié en el miedo y en la estigmatización de las personas migrantes utilizando un conjunto de estrategias discursivas típicas del discurso racista: el peligro de la invasión, el peligro terrorista, la sobrepoblación, la magnificación del fenómeno, el sensacionalismo y la promoción activa de movilizaciones xenófobas.

Ardoval Abreu (2008) ha estudiado la representación de la inmigración en la fotografías de cuatro periódicos canarios (El Día, El Diario de Avisos, La Provincia y Canarias 7) a lo largo de 2007. Los resultados de su estudio evidencian que los medios representan solamente algunos aspectos del fenómeno migratorio: la inmigración se asocia casi en exclusiva a la llegada de embarcaciones (cayucos y pateras) de África, y el inmigrante retratado es casi exlusivamente el "subsahariano" o el marroquí, primando el retrato homogeneizador, en grupo, sobre el que busca la individualidad y la diferencia. Además, el estudio hace hincapié en que el tratamento fotográfico suele identificar a los africanos con la debilidad, la carencia de iniciativa y la subalternidad, se les asocia, además, con el delito, a la vez que se ignora a otros colectivos, más numerosos, como los latinoamericanos. 


\section{Los principales temas relacionados con la inmigración en la prensa de Tenerife}

En el marco de un amplio estudio sobre la percepción de la inmigración y de la convivencia en Tenerife, el Observatorio de la Inmigración de Tenerife ha llevado a cabo un análisis de contenido de los encuadres temáticos en una muestra de 698 titulares y sus correspondientes entradillas. La muestra abarca todos aquellos relacionados con el fenómeno migratorio, publicados en la edición on-line de los tres principales periódicos locales de Tenerife (El Día, Diario de Avisos y La Opinión) a lo largo de tres años (2016-2018).

Estos tres periódicos tienen fuerte implantación y significación en la isla de Tenerife. Todos ellos sufren de forma significativa la crisis económica a partir de 2007, que se traduce en una pronunciada vulnerabilidad empresasarial, que concluyen en cambios de diversa entidad, que fluctúan desde las ventas a grupos nacionales hasta la propia desaparición, como en el caso del diario La Opinión.

El primer aspecto que hay que destacar sobre el tratamiento mediático de la inmigración en Tenerife es que en los últimos tres años la inmigración no es un tema central en la prensa de la isla, especialmente, si lo comparamos con los niveles de atención mediática que despertaba hace una década. En el año 2006, los tres periódicos de referencia han publicado 6.057 noticias sobre la inmigración (Rodríguez Borges, 2010), mientras que en los últimos tres años el numero de noticias publicadas ha sido netamente inferior: 242 en 2016, 98 en 2017 y 358 en 2018.

Hay que tener en cuenta que a lo largo de 2016 llegaron 32.000 inmigrantes a bordo de 588 pateras y cayucos, la inmigración irregular por vía marítima hacia Canarias se transformó en un asunto de alcance europeo y, por primera vez, en el barómetro de opinión del

Gráfico 1 CIS se consideró a la inmigración como la primera preocupación de los españoles (CIS,

Distribución mensual de número de noticias relacionadas con la inmigración en los tres periódicos de referencia

(2016-2018).

Fuente El Aquarius es un barco utilizado por la ONG SOS Méditerraneé para tareas de rescate. OBITen, proyecto Percepciones Intergrupales 2017-2019. estudio 2654). La distribución mensual del número de noticias sobre inmigración a lo largo de los últimos tres años evidencia que hay cuatro repuntes: el primero tiene que ver con el debate europeo relacionado con la "crisis de los refugiados sirios", mientras que los otros tres empiezan con la amplia cobertura mediática de la llegada del Aquarius a España, y están vinculados con la reactivación de la "Ruta Occidental", es decir, con el incremento del número de inmigrantes que han llegado irregularmente a España por vía marítima.

En junio de 2018 el barco, com más de 600 personas a bordo, se encontraba en alta mar tras la negativa de Italia y Malta al atraque en sus puertos. El 11 de junio el Gobierno de Español ofreció el puerto de Valencia para recibir a los inmigrantes.

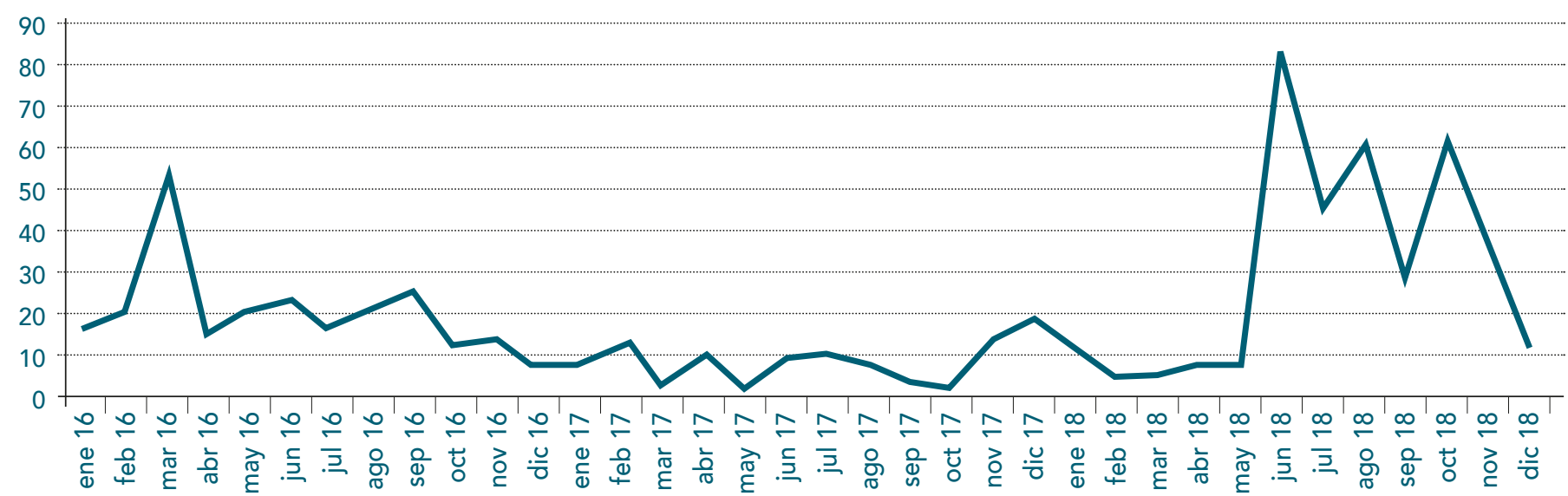


Respecto a los principales temas tratados en las noticias, la inmigración irregular es, con diferencia, el principal tema; le siguen las noticias sobre políticas migratorias que tienen, igualmente, relación prácticamente total con la inmigración irregular. El volumen de noticias mensuales sobre la llegada irregular no es proporcional al numero de personas o embarcaciones llegadas efectivamente al Archipiélago, sino que se incrementa cuando el debate a nivel nacional es más intenso. Es decir, es el debate nacional el que define la agenda mediática sobre la inmigración, más que la realidad migratoria local, aunque esta tendencia puede modificarse si aumenta la intensidad de llegadas irregulares por vía marítima en Canarias.

En 2018, el 38,5\% de las noticias hacia referencia a a la inmigración en las islas, el 32,7\% a la inmigración en España, el 14,0\% al resto de Europa y el 14,8\% al resto del mundo. Como ya subrayaba hace diez años Rodrígez Borges (2010: 21) "con relación al contenido temático de las informaciones sobre inmigración, el comportamiento de los periódicos canarios reproduce, por desgracia, los registros de la prensa nacional. Aunque, afortunadamente, la conexión entre inmigración y delincuencia apenas se percibe".

\begin{tabular}{l|c|c|c|c|}
\hline \multicolumn{1}{c|}{ Temas de las noticias } & $\mathbf{2 0 1 6}$ & $\mathbf{2 0 1 7}$ & $\mathbf{2 0 1 8}$ & Total \\
\hline Inmigración irregular & 31,4 & 39,8 & 51,1 & 42,7 \\
\hline Políticas migratorias e de integración & 14,9 & 14,3 & 20,1 & 17,5 \\
\hline $\begin{array}{l}\text { Solidaridad y defensa de los derechos de } \\
\text { las personas migrantes }\end{array}$ & 17,8 & 22,4 & 5,0 & 11,9 \\
\hline $\begin{array}{l}\text { Acogida de refugiados y menores extranje- } \\
\text { ros no acompañados }\end{array}$ & 13,6 & 2,0 & 9,2 & 9,7 \\
\hline Otros encuadres & 12,0 & 6,1 & 6,7 & 8,6 \\
\hline Análisis y datos sobre inmigración & 7,9 & 12,2 & 5,6 & 7,3 \\
\hline Inmigración como problema & 2,1 & 3,1 & 2,2 & 2,3 \\
\hline Total & 100 & 100 & 100 & 100 \\
\hline
\end{tabular}

Tabla 1

Distribución de las noticias según tema y año (\%).

\section{Fuente}

OBITen, proyecto

Percepciones Intergrupales

2017-2019.

Exceptuando la entrada o permanencia irregular, la vinculación entre inmigración y delitos o con efectos negativos sobre la sociedad canaria es mínima. Por otro lado, llama la atención la invisibilidad de múltiples temas relacionados con la inmigración (la convivencia, la inmigración latinoamericana y europea, los aportes positivos de la inmigración a la sociedad, etc.).

\section{Enfoques securitario, neutral y solidario}

Un aspecto importante para comprender el tratamiento mediático de la inmigración es el tipo de encuadre que se utiliza para presentar las noticias. En los estudios sobre migraciones y medios de comunicación se suele hablar de encuadre securitario, neutral y solidario. El encuadre securitario hace hincapié en los temas relacionados con la seguridad: delincuencia, la presentación de la inmigración como problema, detenciones de inmigrantes irregulares, la gestión de los Centros de Internamiento de Extranjeros, etc. El encuadre solidario, por el contrario, incluye las noticias que resaltan la solidaridad, la defensa de los derechos de las personas migrantes, los proyectos sociales relacionados con la acogida y con la convivencia. En cuanto al encuadre neutral, reagrupa las noticias que reflejan cambios demográficos sin connotarlos ni positivamente ni negativamente, los rescates de personas migrantes y los análisis sobre el fenómeno migratorio. 


\section{Factsheet OBITen_03}

Gráfico 2

Distribución de las noticias según encuadres (\%).

Fuente

OBITen, proyecto Percepciones Intergrupales 2017-2019.
Como se puede apreciar en la siguiente gráfica, el encuadre dominante en la prensa de Tenerife es el neutral, esto se debe sobre todo al tipo de fuentes utilizadas para hablar del principal tema tratado: actualmente en prensa de Tenerife cuando se habla de la inmigración irregular se suele reproducir la información difundidas por las instituciones, es decir, se habla de las operaciones de rescate de personas migrantes llevadas a cabo e informadas por Salvamiento Marítimo.

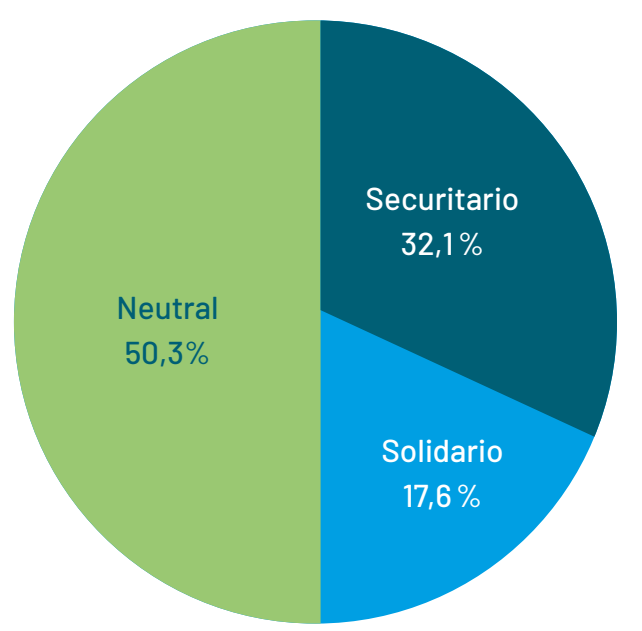

Es llamativa la diferencia entre 2016 y 2018 en relación a los enfoques solidario y securitario: en 2016 el tema al centro de la agenda mediática era la "crisis de los refugiados sirios" que ha tenido un impacto muy limitado en España y nulo en Canarias, y la inmigración irregular en otros países. En este año se publicaron 61 noticias "solidarias"; en 2018, con el aumento de las llegadas por vía marítima de personas migrantes en situación irregular a España, disminuyen las noticias solidarias y aumentan las noticias con encuadre securitario. En otras palabras: se mediatiza el discurso solidario cuando los inmigrantes están lejos, y se redimensiona cuando los inmigrantes llegan a nuestras costas.

\section{Gráfico 3}

Distribución del número de noticias publicas según encuadre y años.

Fuente

OBITen, proyecto Percepciones Intergrupales 2017-2019.

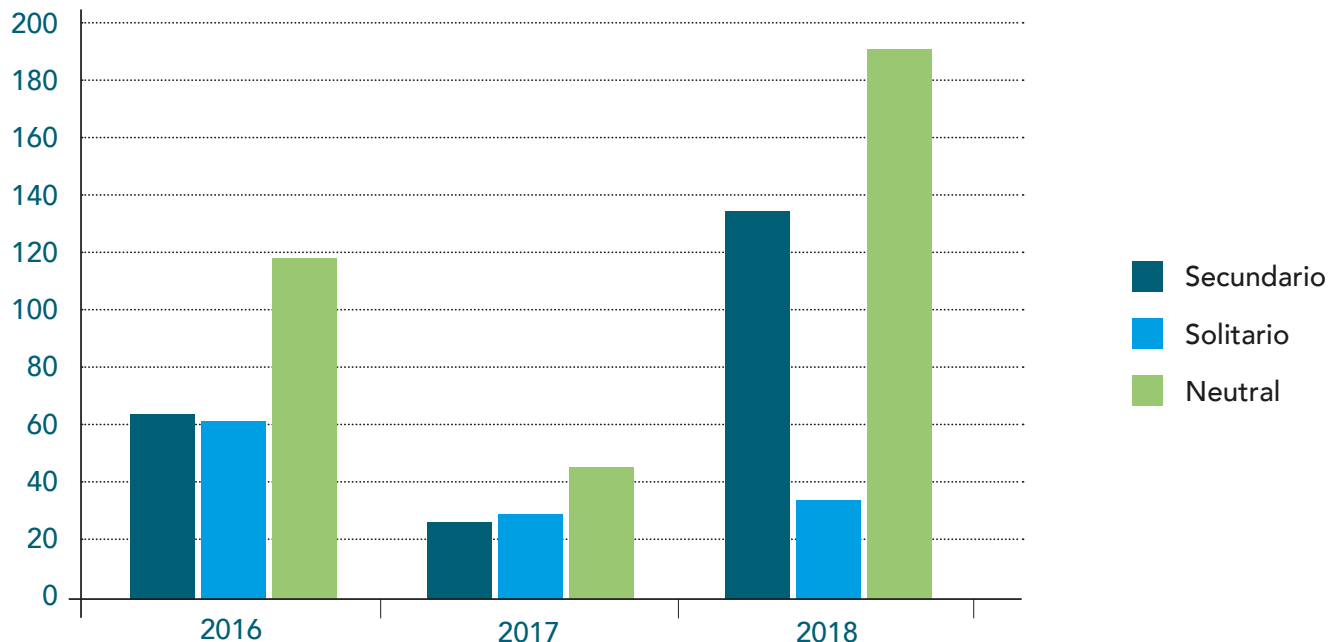




\section{Origen y tipos de inmigrantes que protagonizan las noticias}

El fenómeno migratorio en Tenerife no es homogéneo, sino que varía notablemente según la zona, y el momento. Por ejemplo, actualmente los aspectos más llamativos de los movimientos migratorios que afectan a Tenerife, son el significativo aumento de personas de origen venezolano e italiano. Sin embargo, la prensa sigue sobrerepresentando, como ya hacía hace una década, un perfil específico de inmigrante: africano y en situación administrativa irregular.

En los últimos tres años los inmigrantes de origen africano son el colectivo que protagoniza en mayor medida las noticias sobre inmigración, aunque existen diferencias significativas entre 2016 y 2018. En 2016, las principales noticias estaban vinculadas a la crisis de los refugiados y juntos con las personas de origen africano, numerosas noticas hacían referencias a personas de origen asiático, casi en su totalidad, a personas de origen sirio.

\begin{tabular}{l|c|c|c|c|}
\hline \multicolumn{1}{c|}{ Origen } & 2016 & 2017 & $\mathbf{2 0 1 8}$ & Total \\
\hline África & 38,0 & 54,1 & 70,9 & 57,2 \\
\hline América & 0,8 & 3,1 & 7,8 & 4,7 \\
\hline Asia & 37,2 & 6,1 & 5,3 & 16,5 \\
\hline Europa & 0,0 & 5,1 & 0,3 & 0,9 \\
\hline Canarias & 2,5 & 1,0 & 0,8 & 1,4 \\
\hline No especificado & 21,5 & 30,6 & 14,8 & 19,1 \\
\hline Total & 100,0 & 100,0 & 100,0 & 100,0 \\
\hline
\end{tabular}

Tabla 2

Distribución de los titulares según origen del colectivo que protagoniza la noticia y años (\%).

\section{Fuente}

OBITen, proyecto Percepciones Intergrupales 2017-2019.

Si analizamos los titulares según "tipo de inmigración", el cambio entre los años es aún más significativo: en 2016 se hablaba, sobre todo, de personas refugiadas o solicitantes de asilo, mientras que en 2017 y 2018 disminuyen significativamente las noticias sobre refugiados y aumentan las protagonizadas por inmigrantes irregulares, llegando a representar dos tercios del total de las noticias publicadas en 2018. También en este caso es llamativo que cuando se habla de personas refugiadas, se hable casi exclusivamente de personas de origen sirio, cuando en España y sobre todo en Canarias, el país de origen con un mayor número de solicitantes (10.350 en España) es Venezuela.

\begin{tabular}{l|c|c|c|c|}
\hline \multicolumn{1}{c|}{ Tipo de inmigrantes } & 2016 & 2017 & $\mathbf{2 0 1 8}$ & Total \\
\hline Inmigrantes en general & 9,5 & 27,6 & 14,8 & 14,8 \\
\hline Trabajadores extranjeros & 5,4 & 7,1 & 1,7 & 3,7 \\
\hline Menores Extranjeros No Acompañados & 3,7 & 1,0 & 7,8 & 5,4 \\
\hline Inmigrantes irregulares & 35,5 & 56,1 & 67,9 & 55,0 \\
\hline Refugiados & 40,5 & 6,1 & 5,0 & 17,5 \\
\hline Victimas de explotación sexual & 0,0 & 1,0 & 0,3 & 0,3 \\
\hline Delincuentes & 2,5 & 0,0 & 1,4 & 1,6 \\
\hline Inmigrantes y emigrantes canarios & 2,5 & 1,0 & 0,8 & 1,4 \\
\hline Otros & 0,4 & 0,0 & 0,3 & 0,3 \\
\hline Total & $\mathbf{1 0 0 , 0}$ & $\mathbf{1 0 0 , 0}$ & $\mathbf{1 0 0 , 0}$ & $\mathbf{1 0 0 , 0}$ \\
\hline
\end{tabular}

Tabla 3

Distribución de los titulares según tipo de inmigración que protagoniza la noticia y años (\%).

\section{Fuente}

OBITen, proyecto Percepciones Intergrupales 2017-2019. 
Otro aspecto que hay que destacar, es que la voz de las personas migrantes está prácticamente ausente en las noticias publicadas en los últimos tres años. Las instituciones y los partidos constituyen las fuentes principales de las noticias, mientras que solo en siete noticias de las 698 analizadas, las personas migrantes figuraban como fuente principal, es decir, el $1 \%$. Esta invisibilización de la voz de las personas migrantes ha sido evidencida por numerosos estudios (Van Dijk, 2016; Igartua, Muñiz y Cheng, 2005; Rodríguez Borges, 2010; Fajardo Fernández y Soriano Miras, 2016) y, lamentablemente, es una constante a nivel europeo, nacional y local.

\section{A modo de conclusión}

En los últimos tres años la inmigración ha estado lejos de la atención mediática que se le dedicaba hace una década, si bien a raíz del caso del Aquarius y de la reactivación de la Ruta Occidental, el número de noticias publicadas ha aumentado sensiblemente. En los periódicos publicados en Tenerife existe claro predominio de los temas vinculados a las fronteras marítimas y la entrada irregular de personas inmigrantes. Este encuadre temático incluye noticias sobre la llegada de pateras, la detención de patrones de pateras, el rescate de inmigrantes por parte de Salvamento Marítimo y la muerte de inmigrantes en su intento de llegar a las islas por vía marítima. Un aspecto particularmente importante para comprender la imagen prototípica que la sociedad canaria puede tener de las personas inmigrantes es que, si nos centramos en los colectivos de inmigrantes que protagonizan las noticias, aquellos que están en situación administrativa irregular son citados en dos tercios de las mismas. Estos datos muestran cómo las migraciones marítimas irregulares en los años 2016, 2017 y 2018, a pesar de estar lejos de representar un fenómeno numéricamente significativo, se encuentran en el centro de la agenda mediática cuando se habla de migraciones en Canarias.

En las noticias publicadas en la prensa de Tenerife suele predominar un encuadre neutral, vinculado sobretodo a fuentes de información institucionales. Los titulares en muy pocas ocasiones relacionan explícitamente la inmigración con problemas sociales y, respecto al periodo 2006-2007, los artículos de opinión y las líneas editoriales explícitamente xenófobas y racistas, han desaparecido. Sin embargo, hay que tener en cuenta que a lo largo de 2018, respecto al 2016, ha disminuido sensiblemente el numero de noticias con encuadre solidario (más relacionado con la crisis de refugiados sirios) y ha aumentado el número de noticias con encuadre securitario. 
Para ampliar la información:

Ardévol Abreu, A. (2008). La inmigración, en la fotografía de prensa de Canarias. Revista Latina de Comunicación Social, 11(63), 409-417.

Fajardo Fernández, R. y Soriano Miras, R. M. (2016). La construcción mediática de la migración en el Mediterráneo: ¿no-ciudadanía en la prensa española?. Revista Internacional de Estudios Migratorios, 6(1), 141-169.

Fernández, C. y Corral, A. (2016). La representación mediática del inmigrante magrebí en España durante la crisis económica (2010-2011). Migraciones internacionales, 8(4), 73-103.

Igartua J. J. y Muñiz, C. (2004). Encuadres noticiosos e inmigración. Un análisis de contenido de la prensa y televisión españolas. Zer. Revista de Estudios de Comunicación, 16, 87-104.

Igartua, J. J., Muñiz, C. y Cheng, L. (2005). La inmigración en la prensa española. Aportaciones empíricas y metodológicas desde la teoría del encuadre noticioso. Migraciones, 17, 143-181.

Rodríguez Borges, R. F. (2010). El tratamiento informativo de la inmigración en la prensa de Canarias. Fuentes, temas y enfoques dominantes. Boletín Millares Carlo, (29), 307-330.

Van Dijk, T. A. (2016). Estudios Críticos del Discurso: Un enfoque sociocognitivo. Discurso \& Sociedad, 1, 137-162.

\section{Cómo citar este artículo:}

Buraschi, D. y Fumero de León, I. (2019). La representación del fenómeno migratorio en la prensa escrita de Tenerife. OBITen Factsheet 3-2019. Recuperado de www.obiten.net.

https://doi.org/10.25145/r.obitfact.2019.08

El Observatorio de la Inmigración de Tenerife es una iniciativa conjunta del Cabildo de Tenerife y la Universidad de La Laguna que surge con vocación de estructura permanente y dinámica para avanzar en el conocimiento científico de los movimientos migratorios. OBITen desarrolla su actividad a modo de centro de recopilación, producción y difusión de información que facilita la opinión cualificada y favorece la toma de decisiones que redunden en una mejor gestión del fenómeno inmigratorio y sus implicaciones.

http://www.obiten.net 